\title{
Relación entre IPSS y uroflujometría en pacientes con síntomas urinarios
}

\section{Relationship between IPSS and Uroflowmetry in Patients with Urinary Symptoms}

\author{
Carmen Liliana Fonseca ${ }^{1}$ Carlos Isaías Oliveros ${ }^{1,2,3}$ Rebeca Escobar ${ }^{1,2,3}$ Julián Azuero ${ }^{3}$ \\ Fabián Daza Almendrales 2,3
}

\footnotetext{
${ }^{1}$ Residencia de Urología, Universidad Del Rosario, Bogotá, Colombia

2 Escuela de Medicina y Ciencias de la Salud, Universidad del Rosario, Bogotá, Colombia

${ }^{3}$ Hospital Universitario de la Samaritana, Bogotá, Colombia
}

\begin{abstract}
Address for correspondence Carmen Liliana Fonseca Buitrago, MD, Department of Urology, Hospital de la Samaritana, Bogotá, Colombia (e-mail: carmen.fonseca@urosario.edu.co).
\end{abstract}

Urol Colomb 2019;28:303-306.

\section{Resumen}

\section{Palabras Clave}

- síntomas urinarios del tracto urinario bajo

- uroflujometría

- severidad de los síntomas

- IPSS
Introducción Los cuestionarios clínicos para los síntomas urinarios se diseñaron para objetivar los síntomas urinarios y de esa forma evitar estudios invasivos y costosos, con la división entre síntomas urinarios de vaciado y llenado, sin embargo, los pacientes presentan síntomas que hacen difícil el discernimiento entre obstrucción infravesical, detrusor hipocontractil o incluso detrusor hiperactivo.

Determinar la correlación entre la uroflujometría y la severidad de los síntomas usando el IPSS (International Prostate Symptom Score) en pacientes síntomas urinarios, comparados con urodinamia y determinar su correlación con el diagnostico de obstrucción infravesical, detrusor hipocontráctil o detrusor hipocontráctil

Métodos Estudio retrospectivo en el cual se recolectaron 27 hombres que consultaron por síntomas urinarios de vaciado y asistieron al Hospital Universitario de la Samaritana en Bogotá, Colombia entre abril del 2016 y abril del 2017. El IPSS se realizó a todos los pacientes con el cuestionario autoadministrado. Adicionalmente se realizó uroflujometría y urodinamia con el equipo de urodinámia Medical Measurement System SN077116.

Resultados La edad promedio fue 67,26 años (37-86), IPSS leve del $22 \%$, moderado $59 \%$ y severo $19 \%$, con patrones de uroflujometricos campana $26 \%$, meseta $41 \%$ intermitente $33 \%$, el $37 \%$ presentaron un Qmax menor del 10\%, el cual se consideró obstructivo y una PdetQmax menor de 40 , en un $37 \%$, lo cual es congruente con los resultados de la uroflujometria, además de relacionarse con residuo postmiccional elevado.

Conclusión Existe una correlación entre el IPSS y el Qmax, comparado con patrones urodinámicos, la utilización de los índices como métodos no invasivos para el ahorro de costos si es comparable con hallazgos urodinámicos. Sin embargo, existe un porcentaje no despreciable de pacientes con Qmax menor de 10 que no corresponden a obstrucción infravesical. Es necesario tener en cuenta que hasta el $23 \%$ de los pacientes pueden presentar IPSS moderado o severo sin que el origen sea obstructivo. received

March 25, 2018

accepted

November 29, 2018
DOI https://doi.org/

10.1055/s-0038-1676862.

ISSN 0120-789X.

e ISSN 2027-0119.
Copyright ( 2019, Sociedad Colombiana License terms de Urología. Publicado por Thieme Revinter Publicações Ltda., Rio de Janeiro, Brazil. Todos los derechos reservados.

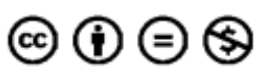




\begin{abstract}
Keywords

- lower urinary tract symptoms

- uroflowmetry

- symptom severity

- IPSS

Introduction Clinical questionnaires for urinary symptoms were designed to perform an objective evaluation of urinary symptoms and thus avoid invasive and expensive studies. However, patients present symptoms that make difficult the discernment between infravesical obstruction and hypocontractile detrusor. The aim of this study is to determine the correlation between uroflowmetry and symptom severity using the IPSS (International Prostate Symptom Score) in patients with urinary symptoms, compared with urodynamics and to determine its correlation with the diagnosis of infra-vesical obstruction or hypocontractile detrusor.

Methods Observational retrospective study in which the clinical records of the patients who consulted for urinary emptying symptoms at La Samaritana University Hospital in Bogotá, Colombia, between April 2016 and April 2017 were reviewed. The IPSS was performed in all patients. Additionally, uroflowmetry and urodynamics were documented with the Medical Measurement System SN077116.

Results A total of 27 patients were included in the study. The average age was 67.26 years. The mild IPSS was found in $22 \%$ of the patients, moderate $59 \%$ and severe $19 \%$. Patterns were identified in uroflowmetry with bell curves in $26 \%$ of patients, plateau $41 \%$ and intermittent in $33 \% .37 \%$ of the patients had a Qmax lower than $10 \mathrm{~mL} / \mathrm{s}$ which was considered obstructive and a PdetQmax less than $40 \mathrm{~cm} \mathrm{H} 2 \mathrm{O}$ in $37 \%$, which is consistent with the results of uroflowmetry, in addition to being related to high postvoid residual volume.

Conclusion There is a correlation between IPSS and Qmax associated with urodynamic patterns. The use of indices as non-invasive methods to reduce costs is comparable with the urodynamic findings; however, there is a non-negligible percentage of patients with Qmax less than $10 \mathrm{~mL} / \mathrm{s}$ who do not correspond to infravesical obstruction. It is necessary to take into account that up to $23 \%$ of patients can have moderate or severe IPSS without the origin of the symptoms being obstructive.
\end{abstract}

\section{Introducción}

Los síntomas urinarios del tracto urinario bajo (SOUB) son comunes en los hombres mayores de 40 años, esos son causados por diferentes patologías. ${ }^{3}$ Los cuestionarios clínicos se diseñaron con el fin de realizar una evaluación objetiva de los síntomas urinarios y de esa forma, evitar estudios invasivos y costosos, ${ }^{1}$ sin embargo, en algunos casos se hace difícil la diferenciación entre obstrucción infravesical y detrusor hipocontráctil. ${ }^{2}$ Hasta el momento, el único estudio que es capaz de discernir entre las diferentes patologías es el estudio flujo presión teniendo mayores costos y siendo invasivo. $^{2}$ La uroflujometría es considerada en la literatura actual como el estudio inicial en SOUB antes de iniciar cualquier tratamiento, con un nivel de evidencia $2 \mathrm{~b}$ y grado de recomendación $\mathrm{B},{ }^{4}$ por lo tanto se consideró realizar este estudio con el fin de conocer el aporte de ese procedimiento no invasivo en el tratamiento que se va a iniciar al paciente, comparándolo con los resultados urodinámicos.

\section{Métodos}

Se realizó un estudio retrospectivo en el cual se revisaron las historias clínicas de hombres que consultaron por síntomas urinarios obstructivos bajos secundarios a crecimiento prostático y asistieron al Hospital Universitario de la Samaritana en Bogotá, Colombia entre abril del 2016 y abril del 2017. El IPSS se realizó a todos los pacientes con el cuestionario autoadministrado. Adicionalmente se realizó uroflujometría y urodinámica con el equipo de urodinámica del Medical Measurement System SN077116.

La severidad de los síntomas se determinó con la escala de IPSS ${ }^{5}$ y se realizó a todos los pacientes con el cuestionario autoadministrado, la uroflujometría y el estudio flujo presión se realizó con el equipo de urodinámica del Medical Measurement System SN077116. La micción se realizó con deseo miccional normal, se utilizaron las urodinámicas que presentaban un volumen mayor de $150 \mathrm{cc}$ hasta 500 cc. Posterior a la uroflujometría se realizó la cistometría y el estudio flujo presión. Se realizó el análisis entre el valor de IPSS divididos entre leves, moderados y severos; el Qmax, volumen evacuado, residuo postmiccional, patrón de flujo y se comparó con los valores urodinámicos PdetQmax y se realizó el cálculo indice de obstrucción.

Se realizó un análisis descriptivo de los datos univariado, reportando para las variables cualitativas, frecuencias $\mathrm{y}$ porcentajes y para las variables cuantitativas medidas de tendencia central y de dispersión de acuerdo a su distribución usando media y desviación estándar para las 
Tabla 1 Características de la Población

\begin{tabular}{|c|c|}
\hline Edad (años) & $67(37-86)$ \\
\hline Residuo postmiccional (mL) & $79,07(0-450)$ \\
\hline \multirow[t]{2}{*}{ Volumen evacuado (mL) } & $273,63(150-500)$ \\
\hline & n (\%) \\
\hline \multicolumn{2}{|l|}{ IPSS } \\
\hline Leve (0-7 puntos) & $6(22)$ \\
\hline Moderado (8-19 puntos) & $16(59)$ \\
\hline Severo (20-35 puntos) & $5(19)$ \\
\hline \multicolumn{2}{|l|}{ Residuo postmiccional } \\
\hline$<10 \%$ & $15(56)$ \\
\hline $10-20 \%$ & $7(26)$ \\
\hline$>20 \%$ & $5(19)$ \\
\hline \multicolumn{2}{|l|}{ PdetQmax } \\
\hline$>60 \mathrm{cmH}_{2} \mathrm{O}$ & $10(37)$ \\
\hline $40-60 \mathrm{cmH}_{2} \mathrm{O}$ & $7(26)$ \\
\hline$<40 \mathrm{cmH}_{2} \mathrm{O}$ & $10(37)$ \\
\hline
\end{tabular}

de distribución normal y mediana y rango intercuartílico para las de distribución no normal. Se utilizó el paquete estadístico SPSS 20.

\section{Resultados}

Se identificaron 27 pacientes que cumplían criterios de inclusión. El promedio fue de 67,26 \pm 30 años (intervalo 37-86) (Tabla 1). La mayoría de los pacientes tenían IPSS moderado (59\%), $22 \%$ leve del $22 \%$, y severo $19 \%$, con patrones de uroflujometría y patrones en la curva de uroflujometría de campana (26\%), meseta $41 \%$ e intermitente $33 \%$.

El 37\% de los pacientes tuvo un Qmax menor del 10\% el cual se consideró obstructivo y una PdetQmax menor de 40, en un $37 \%$.

De los pacientes con un IPSS moderado y severo, el 61\% se encontraban obstruidos, eso se determinó con un Qmax menor de $10 \mathrm{~mL} / \mathrm{s}$, Pdet mayor de $40 \mathrm{~cm} \mathrm{H}_{2} \mathrm{O}$, o un índice de obstrucción mayor que el 40. El 23\% de los pacientes no se encontraban obstruidos y en un $14 \%$ los valores fueron equivocados, por lo tanto se considera que hasta el $23 \%$ pueden cursar con otras patologías que estén asociadas con los síntomas sin que el origen de ella sea la obstrucción. En cuanto a los pacientes con IPSS leves el $83 \%$ no se encontraban obstruidos y el $16 \%$ sí, por lo tanto, a pesar de tener síntomas leves eso no excluye el diagnóstico de obstrucción.

El promedio de volumen evacuado fue $273 \mathrm{cc}$, residuo postmiccional promedio de $79 \mathrm{cc}$, el $37 \%$ de los pacientes no presentó residuo a pesar de que presentó PdetQmax y un índice de obstrucción sugestivo de obstrucción. Un $22 \%$ de los pacientes a pesar de tener curvas anormales y residuos postmiccionales elevados no presentaban un índice de obstrucción ni PdetQmax sugestiva de obstrucción.

\section{Discusión}

Los SOUB pueden presentarse por obstrucción del tracto urinario o hipocontractilidad del detrusor ${ }^{1}$ los cuales se pueden objetivar con la escala de IPSS. La uroflujometría es una herramienta importante utilizada en la mayoría de los pacientes con sospecha de disfunción del tracto urinario bajo. ${ }^{6}$ Aunque algunos pacientes se pueden beneficiar de estudios invasivos, ${ }^{1}$ otros pueden requerir únicamente de ese procedimiento de bajo costo no invasivo, que tiene recomendación de realizarse como primer estudio previo al inicio de tratamiento. ${ }^{4}$ Es importante conocer en nuestro medio el aporte de este estudio y que tipo de tratamiento se debe iniciar, dejando claro que no se puede diferenciar entre detrusor hipocontráctil y obstrucción infravesical. ${ }^{7}$

En nuestro estudio la mayoría de pacientes se encuentran por encima de los 40 años, ${ }^{3}$ con una edad media de 67,26 años. Al igual que en la literatura mundial la mayoría de los pacientes (59\%) presentaban síntomas moderados. El promedio de volumen evacuado es $273 \mathrm{cc}$, comparable con otros estudios similares.

Al ser retrospectivo no se contó con los datos sobre los síntomas específicos del cuestionario IPSS. En los estudios revisados los síntomas más frecuentes son nocturia, aumento de la frecuencia urinaria y vaciamiento incompleto. ${ }^{1}$ La correlación del vaciamiento incompleto con residuo postmiccional mayor del $10 \%$ se relacionó con un índice de obstrucción mayor que el 40.

Se considera por hallazgos del estudio que existe una correlación entre el IPSS y el Qmax, comparado con patrones urodinámicos, además de residuos postmiccionales elevados e índices de obstrucción mayor de 40. La utilización de los índices como métodos no invasivos para la disminución de costos sí es comparable con hallazgos urodinámicos, lo cual apoya la recomendación de solicitarlo previo al inicio de tratamiento, sin embargo, existe un porcentaje de esos síntomas asociado con detrusor hipocontráctil, que en nuestro estudio corresponde a un $22 \%$ requiriendo estudios adicionales, por lo tanto, los estudios flujo presión se deberán realizar en pacientes que no mejoren a pesar del tratamiento.

\section{Conclusiones}

Existe una correlación entre el IPSS y el Qmax, comparado con patrones urodinámicos, además de residuos postmiccionales elevados e índices de obstrucción mayor del 40. Existe un $22 \%$ de los pacientes con síntomas asociado a detrusor hipocontractil quienes requerirán estudios adicionales, valor no despreciable que debe ser tenido en cuenta y comparado con estudios futuros sobre prevalencia de detrusor hipocontráctil.

\section{Responsabilidades Éticas}

Protección de personas y animales. Los autores declaran que para esta investigación no se han realizado experimentos en seres humanos ni en animales.

Confidencialidad de los datos. Los autores declaran que han seguido los protocolos de su centro de trabajo sobre la publicación de datos de pacientes. 
Derecho a la privacidad y consentimiento informado. Los autores declaran que en este artículo no aparecen datos de pacientes.

Financiación

No existió financiación.

Conflicto de intereses

Los autores declaran no tener ningún conflicto de intereses.

\section{Referencias}

1 Oranusi $\mathrm{CK}$, Nwofor AE, Mbonu O. Correlation between international prostate symptom score and uroflowmetry in patients with benign prostatic hyperplasia. Niger J Clin Pract 2017;20(04):454-458
2 Oelke M, Höfner K, Jonas U, de la Rosette JJ, Ubbink DT, Wijkstra H. Diagnostic accuracy of noninvasive tests to evaluate bladder outlet obstruction in men: detrusor wall thickness, uroflowmetry, postvoid residual urine, and prostate volume. Eur Urol 2007;52(03):827-834

3 Parsons BA, Bright E, Shaban AM, Whitehouse A, Drake MJ. The role of invasive and non-invasive urodynamics in male voiding lower urinary tract symptoms. World J Urol 2011;29(02):191-197

4 Gratzke C, Bachmann A, Descazeaud A, et al. EAU Guidelines on the Assessment of Non-neurogenic Male Lower Urinary Tract Symptoms including Benign Prostatic Obstruction. Eur Urol 2015; 67(06):1099-1109

5 Cockett ATK. AYCC. The international consultation on benign prostatic hyperplasia. Scientific International; 1991

6 Singla S, Garg R, Singla A, Sharma S, Singh J, Sethi P. Experience with uroflowmetry in evaluation of lower urinary tract symptoms in patients with benign prostatic hyperplasia. J Clin Diagn Res 2014;8(04):NC01-NC03

7 Salazar MP, Castellanos LT. El estudio urodinámico. Urol Colomb 2014;23(02):128-139 